\title{
10 años de la Educación para el Desarrollo en la Universidad Pablo de Olavide. Tendencias actuales y retos futuros: la Agenda 2030
}

\author{
Guillermo Domínguez-Fernández \\ Esther Prieto-Jiménez \\ Universidad Pablo de Olavide. España. \\ gdomfer@upo.es \\ eprijim@upo.es \\ Luis López-Catalán \\ UNICEF. España. \\ llopez@unicef.es
}

Recibido: 18/5/2019

Aceptado: 6/3/2020

Publicado: 20/7/2020

\section{Resumen}

La Agenda 2030 establece un marco de acción y de cambio para el conjunto de la ciudadanía. El reto de integrar dicha agenda en todos los ámbitos educativos nos lleva a cuestionarnos cómo podemos abordarlos desde la universidad. Por ello, la finalidad del presente artículo es evaluar el impacto que ha ejercido el único máster oficial que se imparte en España en Educación para el Desarrollo cuando se cumplen diez años de su implantación en nuestro país. Debe tenerse en cuenta que dichos estudios persiguen un cambio integral en el alumnado desde el enfoque de la ciudadanía crítica y el empoderamiento en la sociedad en red. Así, presentamos un análisis realizado tomando como población de estudio a los 221 estudiantes que lo han cursado hasta ahora. Los resultados se centran en las tres dimensiones esenciales: progreso académico; empleabilidad, y generación de valores y compromiso. De los datos se concluye que la evolución natural del máster ha alcanzado los tres grandes objetivos inicialmente planteados: formaciónintervención, concienciación-compromiso y acción por el cambio global.

Palabras clave: educación para el desarrollo; universidad; máster oficial; impacto; ciudadanía crítica

Resum. 10 anys de l'Educació per al Desenvolupament a la Universitat Pablo de Olavide: tendències actuals $i$ reptes futurs: l'Agenda 2030

L'Agenda 2030 estableix un marc d'acció i de canvi per al conjunt de la ciutadania. El repte d'integrar aquesta agenda a tots els àmbits educatius ens porta a qüestionar-nos com es poden abordar des de la universitat. Per això, la finalitat del present article és avaluar l'impacte que ha exercit l'únic màster oficial que s'imparteix a Espanya en Educació per al Desenvolupament després de deu anys d'haver-lo implantat aquí. Cal tenir en compte que aquests estudis persegueixen un canvi integral en l'alumnat des de l'enfocament de la ciutadania crítica i l'empoderament a la societat en xarxa. Així, presentem un estudi realitzat prenent com a població d'estudi els 221 estudiants que l'han cursat fins ara. Els 
resultats se centren en les tres dimensions essencials: progrés acadèmic; ocupabilitat, i generació de valors i compromís. De les dades es conclou que l'evolució natural del màster ha arribat als tres grans objectius inicialment plantejats: formació-intervenció, conscienciació-compromís i acció pel canvi global.

Paraules clau: educació per al desenvolupament; universitat; màster oficial; impacte; ciutadania crítica

Abstract. 10 years of development education at the university: Current trends and future challenges in the 2030 Agenda

The 2030 Agenda establishes a framework for action and change for all citizens. The challenge of integrating this agenda in all educational fields has lee us to inquire into how the agenda has been approached in the higher education setting. The aim of this article is to evaluate the impact that the only official master's degree in education for development in Spain-now in its tenth 10 consecutive year-has had in achieving a comprehensive change in students from the perspective of critical citizenship and empowerment in the networked society. We present a study carried out with a population comprising the 221 students who have completed the degree. The results focus on the three essential dimensions: academic progress, employability and the promotion of values and commitment. Based on the data, it is concluded that the natural evolution of the master's degree has achieved the main objectives it initially set out to achieve: training, intervention, awareness-raising, commitment and action for global change.

Keywords: education for development; university, official master; impact; critical citizenship
Sumario
1. Introducción
2. El modelo de la ciudadanía crítica inclusiva y el empoderamiento en las redes sociales en el marco de las grandes metas
4. Presentación y discusión de los datos obtenidos
5. Conclusiones
Referencias bibliográficas

3. Métodos, instrumentos y muestra

\section{Introducción}

La Agenda 2030 (ONU, 2015) y las grandes metas que se propone alcanzar con los 17 objetivos de desarrollo sostenible (ODS) se convierten en un referente a considerar en cualquier proceso educativo actual. Centrándonos en el ámbito universitario, la declaración de Bolonia (2009) ya lo revolucionaba, puesto que basaba la formación de grados y postgrados en el desarrollo de competencias y buscaba el equilibrio entre capacidades, conocimientos, habilidades y actitudes. Si unimos estas dos coordenadas, encontramos que la institución universitaria debe asumir la responsabilidad de ofrecer una formación integral de los ciudadanos. Ante este compromiso, en el año 2010 un grupo de docentes del Grupo de Investigación de Educación de la Universidad 
Pablo de Olavide, de Sevilla (GEDUPO), con el apoyo institucional de la Universidad, promueve la creación del primer máster en Educación para el Desarrollo, Sensibilización Social y Cultura de Paz, preocupado por movilizar y promover los objetivos de desarrollo del milenio (ODM). La temática central del máster, la Educación para el Desarrollo (EpD), encuentra a su vez respaldo en declaraciones, tratados y políticas sociales, tanto a nivel internacional (The Maastricht Global Education Declaration, 2002; UNESCO, 2015) como a nivel nacional (Ley Española de Cooperación Internacional para el Desarrollo (LECI) (1998), en los planes directores de la cooperación española (AECID, 2009 y 2018), en la estrategia de EpD de la Agencia Española de Cooperación Internacional (Ortega, 2007) y, finalmente, en el marco autonómico de Andalucía, en el Plan Andaluz de Cooperación Internacional para el Desarrollo (AACID, 2015 y 2018).

La finalidad inicial que se marcó el máster se centraba en tres aspectos esenciales: formar y especializar al alumnado en base a los conocimientos propios de la educación para el desarrollo, dotarle de herramientas y recursos necesarios para el desempeño profesional y, fundamentalmente, el desarrollo personal, mediante la adquisición de valores, concienciación, compromiso e implicación activa, así como su transferencia a la práctica.

La evolución del máster ha tenidos tres etapas bien diferenciadas:

- 1. a fase. Puesta en marcha (2010). El máster surge en el contexto de la cultura de lucha por el $0,7 \%$ y con el auge de las organizaciones no gubernamentales para el desarrollo (ONGD), identificando en ellas un yacimiento de empleo futuro.

- 2. a fase. Crisis y consolidación (2014). La llegada de la crisis (económica, social, política...) afectó directamente a este máster. La reducción de las políticas sociales incumbía tanto a la cultura de lucha por el $0,7 \%$ como a la dificultad del alumnado por acceder económicamente a títulos de postgrado. Esto demandó un cambio de estructura, de módulos y de profesorado, y se centró más en el compromiso y en la motivación de los docentes, lo que fortaleció la plantilla.

- 3. a fase. Replanteamiento y renovación (2018). Etapa actual, en la que, tras la última renovación de la titulación, se identificaron necesidades de actualización atendiendo a las nuevas exigencias y a los nuevos perfiles demandantes. Actualmente el alumnado procede principalmente de titulaciones de Ciencias de la Educación y Ciencias Sociales, con un alto nivel académico y un valor añadido de compromiso e implicación con el entorno. Por ello se está potenciando la relación con las ONGD, seleccionándolas por la calidad de su funcionamiento como eje del máster y base del futuro desempeño profesional.

En este trabajo evaluaremos el aprendizaje y las competencias adquiridas por el alumnado, su transferencia a la empleabilidad y la adquisición de valores y actitudes de compromiso activo y ciudadanía crítica, atendiendo a 
los tres objetivos y dimensiones esenciales (la académica, la profesional y, sobre todo, la personal), lo que servirá como evidencia para validar el proceso de cambio y renovación del máster en el marco de la Agenda 2030 (ONU, 2015).

\section{El modelo de la ciudadanía crítica inclusiva y el empoderamiento en las redes sociales en el marco de las grandes metas}

La concepción en la que se fundamenta nuestra investigación parte de un modelo tridimensional propio en el que se consideran tres vectores: los modelos de desarrollo y cooperación; los modelos educativos, y los modelos de educación para el desarrollo.

El primer vector estudiado se centra en los modelos de desarrollo y cooperación en base a los planteamientos de autores como Boni (2010), quien, a su vez, parte de la clasificación de los modelos económicos de Vidal (1995), Bustelo (1998), Sanahuja (2001) y Alonso y Sanahuja (2007), los cuales diferencian claramente cinco modelos:

a) Décadas de 1950 y 1960, comienza la cooperación para el desarrollo. Es el final del imperialismo, las inversiones perdidas y el inicio del desarrollismo. La cooperación por bloques.

b) Década de 1970, denominada la persistencia de la desigualdad, con la cooperación en base a las necesidades básicas, la crisis de 1973 y el nuevo orden internacional.

c) Década de 1980, con la crisis de la deuda y la denominada década perdida, con el comienzo del neoliberalismo, el derecho al desarrollo y la aparición de una cooperación con «rostro humano» (derechos).

d) Década de 1990, con el final de la guerra fría, el desarrollo de los paradigmas del desarrollo humano y el comienzo de los procesos de globalización, con la aparición de conceptos como sostenibilidad, género y equidad, que culminan con la noción de solidaridad y la aparición de los OMD en el año 2000.

e) En estos momentos hemos entrado en una etapa, según Boni (2010), en la que se cuestiona la eficacia de la ayuda y se busca, cada vez más, la «ayuda inclusiva» a los grupos más desfavorecidos, con la intención de conseguir una mayor transparencia y más impacto.

Finalmente, Groves y Hinton (2004) concluyen hablando de la existencia de tres grandes modelos o paradigmas de desarrollo y cooperación: asistencialista y de donación; de participación y construcción del partenariado, y de desarrollo basado en los derechos y el empoderamiento.

La segunda coordenada de nuestro estudio se centra en los modelos y paradigmas de educación desarrollados por Shulman (2019), Bolívar (2007) y Pérez y Pérez (2013), adaptados a este campo por Domínguez, Prieto y Álvarez (2019). Teniendo en cuenta estas propuestas y haciéndolas convergentes con 
la clasificación que acabamos de desarrollar de los modelos de cooperación al desarrollo, tendremos en cuenta los siguientes:

a) Modelo artesanal-academicista (aprender), antes de la década de 19501960, vinculado al modelo asistencialista.

b) Modelo conductista (aprender a hacer), unido al desarrollismo de la década de 1970.

c) Modelo humanista (aprender a ser), en la década de 1980, unido al movimiento crítico y solidario.

d) Modelo cognitivista y constructivista (aprender a aprender), en la década de 1990, unido a la ciudadanía global y al crecimiento sostenible.

e) Modelo sociocrítico (aprender a transferir), en las décadas de 1990 y 2000, uniendo las grandes metas a la ciudadanía global y a la emancipación.

f) Hoy podríamos hablar más de modelos holísticos que integran varias tendencias, sobre todo la cognitivista y la sociocrítica, afianzando cada vez más el movimiento de la ciudadanía global, la ciudadanía crítica y el empoderamiento de la educación a partir de las redes sociales (aprender a desaprender en la línea de Covey, 1998).

El último segmento estudiado es la aplicación de los modelos de desarrollo a la educación, con lo que surgieron los modelos de educación para el desarrollo. Tradicionalmente, en este campo han sido llamados generaciones (Mesa, 2000, basada en Gómez y Sanahuja, 1999). Esta autora plantea las cinco generaciones siguientes:

1. Caritativa o asistencial.

2. Desarrollista.

3. Crítica y solidaria.

4. Desarrollo humano y sostenible.

5. Ciudadanía global.

Posteriormente ha habido otras aportaciones claves para entender la evolución de la EpD (Argibay y Celorio, 2005) que reducen a cuatro los enfoques (asistencial, crítico y solidario, educación global y educación para la ciudadanía global), y Celorio (2014), que retoma las cinco generaciones (beneficencia, desarrollista, desarrollo sostenible, movimientos populares y de ONGD altermundistas), otorgando una importancia especial al contexto de la educación emancipadora. Recientemente, Mesa (2014) incide en la necesidad de replantearnos, en el contexto actual, algunos aspectos: la dialéctica global y local, la relación Norte-Sur, la solidaridad ante los desequilibrios (brechas), el uso de las nuevas tecnologías y las redes sociales en los ámbitos socioeducativos, la educación transformadora y la mayor complejidad de las situaciones, que invitan a dar otra perspectiva a la acción en este campo. Todo ello, unido a lo ya manifestado en el Congreso de Bilbao (Celorio y López de Munain, 2007) y que sigue siendo tendencia en las reuniones científicas actuales, hace necesario 
dar un paso adelante y aludir a modelos o incluso, en algunos aspectos, a paradigmas de EpD.

Ante este reto, fundamentamos el posicionamiento del presente artículo definiendo un nuevo enfoque de Educación para el Desarrollo, considerando el contexto de la cibersociedad (Jones, 2003) y una dimensión de la EpD más crítica y emancipadora, para el empoderamiento con las redes sociales a favor de la educación inclusiva y la incorporación a la dialéctica en esta lucha de los grupos desfavorecidos (Prieto-Jiménez, Domínguez-Fernández y López-Catalán, 2018).

Figura 1. Una visión tridimensional de desarrollo, educación y EpD. 6 enfoques

\begin{tabular}{|c|c|c|c||}
\hline \multicolumn{7}{|c|}{ UNA VISIÓN TRIDIMENSIONAL DE DESARROLLO, EDUCACIÓN Y EpD: } \\
6 ENFOQUES
\end{tabular}

Fuente: elaboración propia. 
Este nuevo modelo propuesto se ubica en el contexto de las grandes metas (la Agenda 2030 y los ODS), la cibersociedad (Jones, 2003) y la cuarta revolución tecnológica, la 4.0 (big data, etc.). Este marco nos lleva a la necesidad de realizar una reorganización en función de las redes sociales (ciberorganizaciones), para lo que es necesario un ciudadano crítico y activo, que persiga la inclusión y luche contra las brechas tecnológicas y sociales, empoderándose y empoderando mediante la oportunidad de las redes sociales. Todo ello desde una perspectiva transversal de temas claves para el cambio, como son el clima, el género, el consumo responsable, el comercio justo, la sostenibilidad, el desarrollo de lo local en lo global y viceversa, etc. (Morón, López y Cobos, 2017).

Un ejemplo de este nuevo modelo de empoderamiento donde, mediante las redes sociales, se pueda participar en la lucha por los desequilibrios y las situaciones injustas, independientemente de las características socioeconómicas. Se trata de movimientos tales como el 15M, Me Too, Fridays for Future, la Primavera Árabe o las grandes ONGD de lucha, como Open Arms, Médicos sin Fronteras, Greenpeace y su influencia a nivel internacional de problemas locales con impacto global.

\section{Métodos, instrumentos y muestra}

Siendo la finalidad de este estudio evaluar el impacto que ha ejercido el máster en EpD en sus diez años de vida, basamos nuestra metodología en un estudio mixto donde «se recogen y se analizan datos, se integran hallazgos y se formulan inferencias utilizando aproximaciones o métodos cuantitativos y cualitativos en un mismo estudio o programa de investigación" (Teddlie y Tashakkori, 2009, p. 4). Si bien es eminentemente cualitativo, nos apoyamos en la flexibilidad del método mixto, articulando perspectivas cuantitativas (cuestionarios) y cualitativas (entrevistas) (López-Roldán y Fachelli, 2015). La primera fase del estudio fue la de conceptualización, donde sentamos las bases de la investigación; la segunda, la de diseño y aplicación de los instrumentos y los análisis de datos, y la tercera, la de explicación y comprensión de toda la información trabajada, con la intención de generar conocimiento (Teddlie y Tashakkori, 2009).

Por ello diseñamos un sistema de categorías a priori que nos sirvió de base tanto para el cuestionario como para la entrevista individualizada. Fueron las siguientes: perfil del alumnado, motivación inicial, evolución durante el máster, impacto final del máster en el alumnado y propuestas de mejora. El cuestionario consta de quince preguntas. Las cinco primeras son de identificación, a continuación encontramos tres escalas de estimación referentes a «antes de cursar el máster», «durante el máster» y "al finalizar el máster», y, para terminar, cinco preguntas abiertas de opinión.

La entrevista pretendía validar toda la información recabada mediante los cuestionarios, por lo que se articuló en diez preguntas de carácter abierto, apoyándose en las cuatro categorías base del cuestionario: «antes de cursarlo», 
«durante el máster», «al finalizarlo» y "propuestas de mejora». Con la entrevista el alumnado seleccionado aclaró, matizó y confirmó lo recogido en el cuestionario.

Ambos instrumentos fueron validados con el método Delphi de consulta de expertos.

El número de estudiantes que han cursado el máster en esta década asciende a 221. Aplicando un nivel de confianza del $95 \%$ y un error muestral del $5 \%$, obtuvimos una muestra representativa de 140 antiguos alumnos a los que se les aplicó el cuestionario. El muestreo de la entrevista se realizó a modo de conveniencia. Se eligió a un representante de cada una de las promociones y se llegó a un total de nueve entrevistas en profundidad. En la tabla 1 presentamos el perfil de los participantes en esta investigación.

Tabla 1. Datos de los participantes en los cuestionarios

\begin{tabular}{|c|c|c|c|c|c|}
\hline \multicolumn{2}{|c|}{ Sexo } & \multicolumn{2}{|c|}{ Edad } & \multicolumn{2}{|c|}{ Titulación de acceso } \\
\hline Mujer & $75 \%$ & $\begin{array}{l}\text { Menos de } 25 \\
\text { años }\end{array}$ & $6,25 \%$ & Educación Social & $12,50 \%$ \\
\hline \multirow[t]{6}{*}{ Hombre } & $25 \%$ & 26-30 años & $43,75 \%$ & Educación y Trabajo Social & $8,30 \%$ \\
\hline & & 31-35 años & $25,00 \%$ & Magisterio & $41,70 \%$ \\
\hline & & 36-40 años & $16,70 \%$ & Pedagogía & $4,15 \%$ \\
\hline & & $\begin{array}{l}\text { Más de } 40 \\
\text { años }\end{array}$ & $8,30 \%$ & Psicología & $14,60 \%$ \\
\hline & & & & Trabajo Social & $6,25 \%$ \\
\hline & & & & Otras titulaciones* & $12,50 \%$ \\
\hline
\end{tabular}

* En «Otras titulaciones» incluimos Ciencias Ambientales, Enfermería, Física, Periodismo, Química y Sociología.

Fuente: elaboración propia.

La elección de los sujetos para realizar las entrevistas se basó en los siguientes criterios: representación por promoción académica, variedad de sexo, edad e itinerarios cursados (profesionalizante o investigador). El resultado se muestra en la tabla 2.

Tabla 2. Datos de los participantes en las entrevistas

\begin{tabular}{|c|c|c|c|c|c|c|c|c|c|c|}
\hline & \multicolumn{2}{|c|}{ Sexo } & \multicolumn{5}{|c|}{ Edad } & \multirow{2}{*}{$\begin{array}{c}\text { Promoción } \\
\text { Curso }\end{array}$} & \multicolumn{2}{|c|}{ Itinerario } \\
\hline & $\mathrm{H}$ & M & $<25$ & $26-30$ & $31-35$ & $36-40$ & 40 & & Profes & Invest. \\
\hline E1 & & $x$ & & & & & $x$ & $2010-2011$ & & $x$ \\
\hline E2 & & $x$ & & & & $x$ & & 2011-2012 & $x$ & \\
\hline E3 & & $x$ & & & $x$ & & & $2012-2013$ & $x$ & \\
\hline E4 & & $x$ & & $x$ & & & & 2013-2014 & $x$ & \\
\hline E5 & $x$ & & & $x$ & & & & 2014-2015 & & $x$ \\
\hline E6 & & $x$ & & $x$ & & & & $2015-2016$ & & $x$ \\
\hline E7 & $x$ & & & & $x$ & & & $2016-2017$ & & $x$ \\
\hline E8 & & $x$ & $x$ & & & & & $2017-2018$ & & $x$ \\
\hline E9 & $\mathrm{x}$ & & $x$ & & & & & 2018-2019 & $x$ & \\
\hline
\end{tabular}

Fuente: elaboración propia. 
El tratamiento de los datos cuantitativos se realizó mediante el SPSS.25, y los datos cualitativos se categorizaron con el apoyo del atlas.ti 8.1. Al tratarse esta de la primera publicación resultante del estudio, los datos que se presentarán han sido tratados únicamente con la estadística descriptiva (frecuencias y porcentajes).

\section{Presentación y discusión de los datos obtenidos}

Para la presentación de los datos atenderemos a las tres dimensiones principales que venimos trabajando en este artículo: el perfil y la motivación del alumnado; el impacto del máster en el desarrollo profesional, y fundamentalmente el desarrollo personal.

\subsection{Perfil de los participantes y motivación para cursar el máster}

Atendiendo a la base de datos del centro de estudios de postgrado, las características básicas de los alumnos demandantes del máster nos indican que sus edades están comprendidas entre los 26 y los 35 años. De ellos el 43,75\% tiene entre 26 y 30 años, lo que indica que están complementando su formación y abiertos a definir su trayectoria profesional. El otro $25 \%$, situado entre los 30 y 35 años, lo hace para consolidar su trayectoria y/o abrirse nuevas perspectivas laborales. Hay un 25\% que representa a los mayores de 35 años. En la mayoría de los casos se trata de estudiantes que han retomado su proceso de formación.

En cuanto a los perfiles académicos predominan los egresados de Ciencias de la Educación $(45,8 \%)$ con posibilidades de definir su próxima trayectoria profesional y proclives a un nuevo campo de acción. Posteriormente hallamos, con un 27,1\%, los procedentes de Ciencias Sociales (Educación Social, Trabajo Social), seguidos de un 14,6\% de Psicología. Finalmente contamos con un $12,5 \%$ procedente de otras titulaciones, que, al ser tan diversas, marcan uno de los valores añadidos del máster. La heterogeneidad de niveles, intereses y perspectivas consolidan la interdisciplinariedad y la riqueza del grupo, siendo este el gran reto en el proceso de enseñanza-aprendizaje.

Para concluir con el estudio del perfil del alumnado del máster, atendemos al factor motivacional que dio acceso a la titulación.

Considerando los datos previos, podemos identificar a tres grupos de participantes claramente definidos:

a) Expertos en el ámbito de las ONGD, tanto a nivel nacional como internacional. Aun teniendo una base práctica considerable, la han complementado con teoría, herramientas y recursos, así como estrategias válidas, tanto para la intervención como para la gestión organizacional, la evaluación o la investigación. Su paso por el máster les ha llevado incluso a realizar la tesis doctoral y están sirviendo de referencia, tanto en la universidad como en el sector de las ONGD. Este perfil se corresponde con educadores y 
Figura 2. Factor motivacional del alumnado para cursar el máster

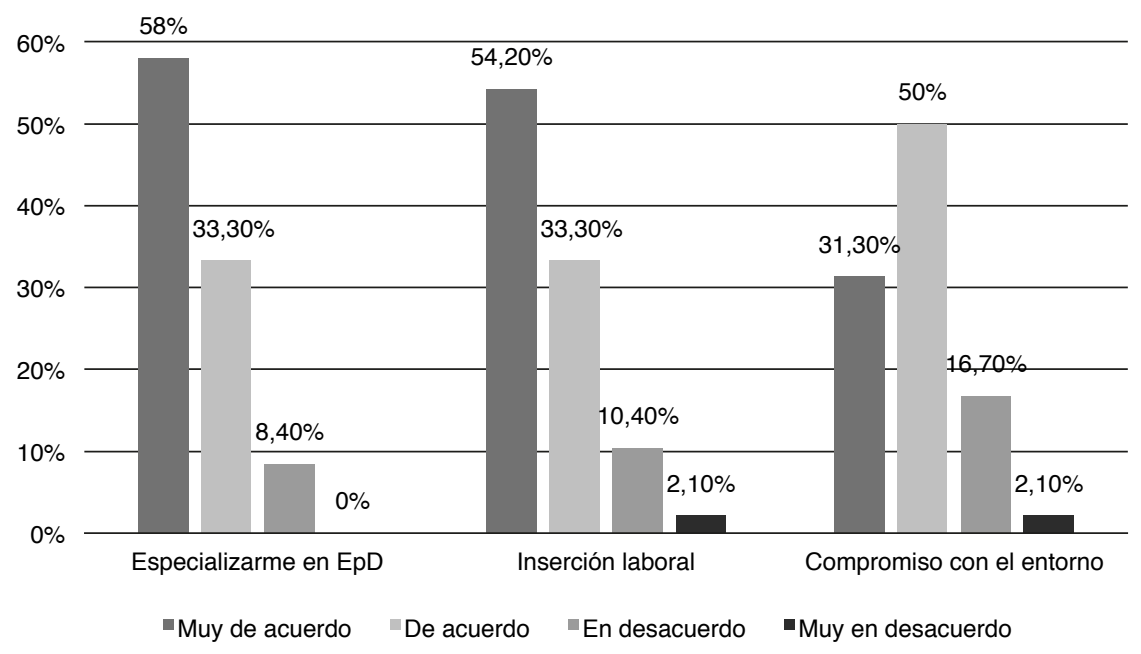

Fuente: elaboración propia.

expertos de otras características, con responsabilidad en ONGD que vinculan conocimiento con realidad.

b) Alumnado que no tenía experiencia en $\mathrm{EpD}$, pero con un alto grado de preocupación por los problemas mundiales y que manifiesta una concienciación que desea incorporar en su futuro desempeño profesional. Este es el grupo mayoritario que encontramos en todas las ediciones y se trata del dinamizador del ambiente del grupo. Se corresponde con un perfil que en general está formado por educadores y maestros. Su nivel de conocimiento de la EpD es escaso o nulo, pero su grado de compromiso y de concienciación es alto, por lo que el máster les dotó de los contenidos y de las herramientas necesarios, y en la actualidad se encuentran transfiriendo lo aprendido en centros educativos, en la dirección y gestión de organizaciones o como técnicos de referencia en las ONGD.

c) Finalmente, un grupo de personas que acceden al máster para obtener una certificación que les haga obtener mayor puntuación en la mayoría de los casos para las oposiciones, especialmente de educación. Tras su paso por el máster descubren una nueva dimensión y una misión distinta de la educación, por lo que integran la EpD en su propio perfil docente, además del cambio que experimentan a nivel personal. Aquí conseguimos el efecto multiplicador, puesto que hemos formado a futuros formadores que, aunque llegaron al máster con baja motivación o concienciación por la EpD, egresan con un alto nivel de conocimiento, concienciación y motivación por integrarla en su práctica docente. 
Tras analizar el perfil y la motivación del alumnado, pasamos a identificar el impacto que el máster ha tenido en cuanto a su desarrollo profesional.

\subsection{Impacto del máster en el desempeño profesional}

Abordaremos este apartado dividiendo la información, atendiendo primeramente a la inserción laboral del alumnado y en segundo lugar a lo relativo a la carrera investigadora. Ambos aspectos son claves en el desempeño profesional del alumnado.

\subsubsection{La empleabilidad tras cursar el máster}

$\mathrm{Si}$ atendemos a los últimos datos facilitados por el Observatorio ARGOS (2016) perteneciente al Servicio Andaluz de Empleo, encontramos que este máster cuenta con un alto porcentaje de inserción laboral en comparación con titulaciones del ámbito socioeducativo.

Como actualización de esos datos nos ceñimos a la información recabada en este estudio, donde se aprecia que la gran mayoría de egresados del máster actualmente se encuentran empleados $(83,3 \%)$, matizando que dentro del $16,7 \%$ que aún no está trabajando hallamos alumnado de las últimas promociones.

Figura 3. Empleabilidad de los másteres del ámbito socioeducativo en la UPO

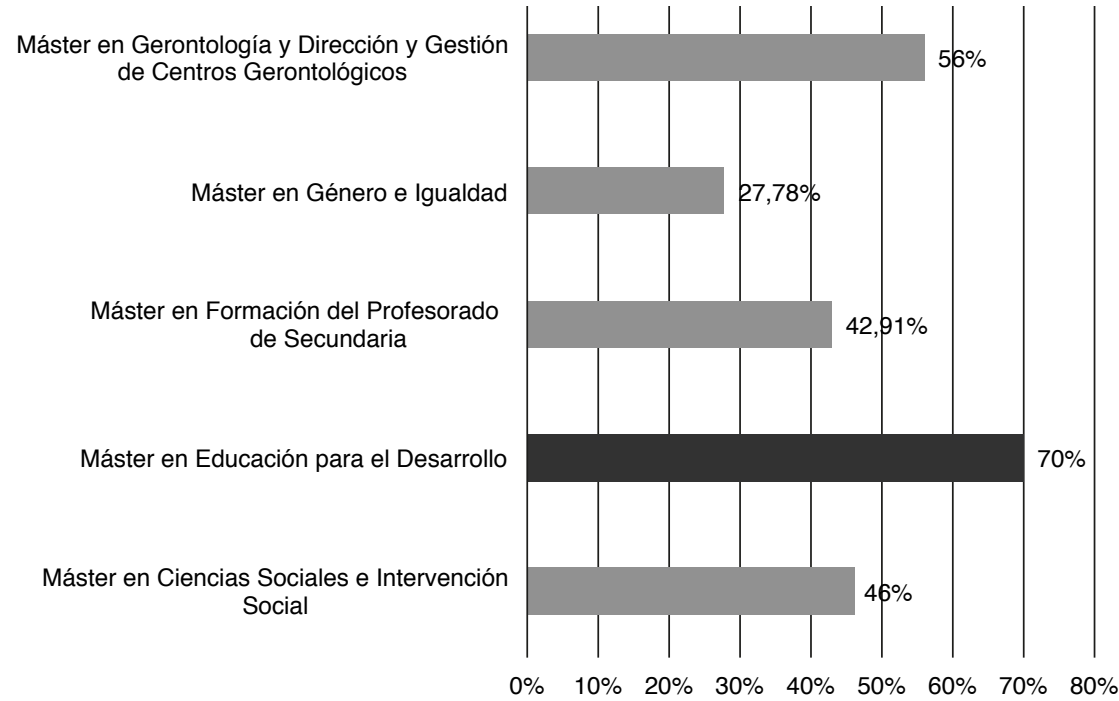

Fuente: elaboración propia. 
Figura 4. El desempeño profesional en el alumnado del máster

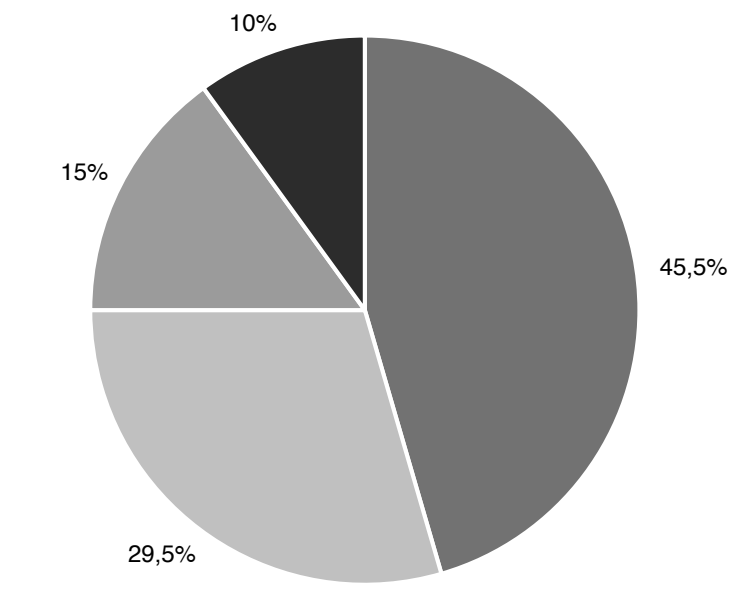

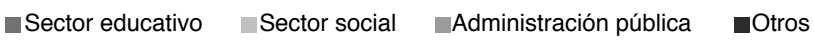

Fuente: elaboración propia.

La gran mayoría desempeña su profesión en el sector educativo (un $45,5 \%$ ), donde destacan los que trabajan en la educación primaria, seguidos de los profesores de secundaria y los orientadores o los que desempeñan su labor en la universidad, así como los educadores que se encuentran en ámbitos que sufren riesgo de vulnerabilidad y de exclusión. En cuanto al sector social (un 29,5\%), destacan los técnicos y los directivos en ONGD y en instituciones sociales especializadas en cuestiones de género, adicciones, movilización social, participación ciudadana y salud, entre otras. Otro ámbito de relevancia es el de la Administración pública (un 15\%), donde desempeñan cargos tales como responsables de áreas sociales y de cooperación en ayuntamientos, en universidades populares, en agencias de cooperación, en consulados, además de gestión en universidades. Finalmente, un $10 \%$ de ellos se encuentran empleados en sectores alejados a lo estudiado en el propio máster y que se fundamentan en el sector servicios (restauración, comunicaciones, etc.).

\subsubsection{El impacto del máster en el mundo de la investigación: tesis doctorales} La segunda dimensión que abordamos en el impacto de desempeño profesional del máster se centra en la puerta de entrada al mundo de la investigación y en la culminación de la tesis doctoral como otra vía de empleabilidad de nuestro alumnado.

En nuestra década del máster se han leído doce tesis doctorales tras iniciarse en el itinerario investigador. Como hemos indicado anteriormente, el pro- 
ceso de Bolonia obligaba a realizar un título de maestría para acceder a diferentes doctorados oficiales. Esto sirvió de reclamo en las primeras ediciones, donde se concentra el 90\% de las tesis anteriormente indicadas que se han defendido en el marco de este máster.

En la segunda etapa se congeló el proceso de defensa y, finalmente, en esta tercera etapa, el alumnado ha recuperado el interés por la investigación, ya que en la actualidad se han leído dos tesis y hay nueve en período de realización.

Igualmente contamos con egresados del máster que son docentes en diferentes universidades (Ávila, Sevilla, UPO, Huelva, Málaga, México, Perú, UNAN-Managua, UNED, etc.), al igual que participan como docentes en el propio título de postgrado.

\subsection{El impacto del máster en el desarrollo personal}

Finalmente, el último punto que abordaremos es el desarrollo personal que el máster ha generado en los estudiantes. En base a los objetivos iniciales de la titulación, el cambio de visión del alumnado, la concienciación, el fomento del compromiso y la acción eran nuestra mayor misión. Comprobamos la opinión del propio alumnado al respecto.

En la nube de términos anterior se evidencia claramente que se han conseguido los objetivos que pretendíamos: la triple dimensión de formación, empleabilidad y cambio personal.

En cuanto a la dimensión académica destaca el término conocimientos, que hace hincapié en la formación extra y en la ampliación de saberes, que finali-

Figura 5. ¿Qué te aportó el máster?

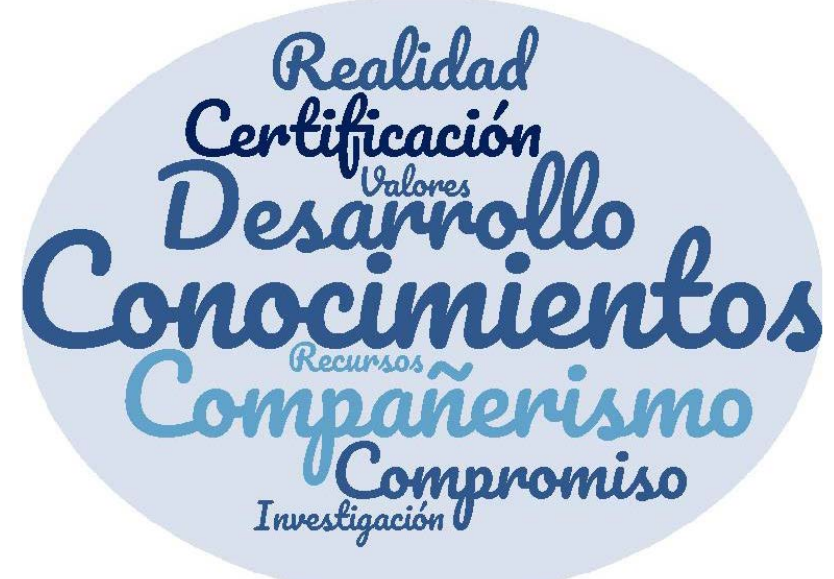

Fuente: elaboración propia. 
za en una certificación que les acredita tanto para ampliar su currículum como para sumar méritos con vistas a una oposición y a la inserción laboral.

Relacionado con la empleabilidad incluimos recursos, que aluden a herramientas y técnicas para desarrollar esta materia en su entorno laboral, e investigación, que identifica el máster como una puerta de acceso a este mundo y que culmina con la realización de la tesis doctoral.

La dimensión personal se recoge con el término realidad, que incluye tanto un cambio de visión propia del mundo con un carácter más global y crítico, como un proceso de concienciación. Le seguiría desarrollo, donde el alumnado identifica dos aspectos claves de evolución, tanto profesional como personal. Dentro de esta dimensión también consideramos compromiso y valores, matizando que su paso por el máster les ha hecho cambiar internamente. Finalmente ha resultado muy recurrente el hecho de la creación de redes de amigos y compañeros (compañerismo) como una de las aportaciones más relevantes y duraderas de los estudios de postgrado.

Tal y como pretendíamos, el alumnado interpreta el impacto del máster de una manera más marcada en la dimensión personal, aunque también valora sus aspectos académico y laboral.

\section{Conclusiones}

Tras estos diez años de experiencia del máster de Educación para el Desarrollo en la Universidad Pablo de Olavide hemos ido consolidando la titulación, que ha pasado de peligrar su activación en ciertas ediciones por no llegar a las quince matrículas a tener, en la última edición, un total de 198 solicitudes. Seguimos siendo el único máster de esta temática a nivel nacional. Así, su porcentaje de alumnado tanto del ámbito nacional como internacional ronda el $20 \%$, lo que denota un indicador de calidad e impacto.

Considerando los tres objetivos que nos planteamos desde el principio y que se han ido enriqueciendo con la revisión de los modelos, las generaciones y el posicionamiento propio, podemos deducir las conclusiones que indicamos a continuación.

Respecto a la formación del alumnado se está consiguiendo cambiar su mentalidad. Inicialmente los estudiantes consideraban el máster de Educación para el Desarrollo como una mera certificación, pero se les hizo ver el valor añadido que poseía dicha cualificación acerca de competencias en tres perfiles: educadores, técnicos y directivos de las organizaciones del ámbito de la EpD. La mayoría de los estudiantes proviene de Magisterio y de perfiles sociopsicológicos y educativos, y su objetivo es el de conseguir certificación para mejorar su currículum (fundamentalmente con vistas a las oposiciones), pero finalmente concluyen su formación considerándola como una especialización y una cualificación de sus titulaciones iniciales. Identifican esta especialización imprescindible en su desarrollo profesional, no habiendo sido tratada en ningún momento en su formación en los grados, lo que les lleva a generar nuevas expectativas. Esto se debe al proceso de estudio del perfil del alumnado, que 
nos ayudó a planificar y a adaptar la actuación educativa de manera eficaz (Garrote y Jiménez, 2018). Tras estas adaptaciones, el alumnado es capaz de abarcar las tres dimensiones (académica, personal y laboral), potenciando su flexibilidad y su cambio de posicionamiento ante nuevas situaciones. Esto nos permite trabajar en nuestro modelo de $\mathrm{EpD}$ de ciudadanía crítica inclusiva y de empoderamiento mediante las redes sociales.

El segundo objetivo del máster se centraba en conseguir una mayor concienciación y un grado más elevado de compromiso. Considerando nuestro posicionamiento de partida, este cambio tendría que girar en torno al desarrollo de una ciudadanía crítica, emancipadora e inclusiva, rompiendo con las brechas tecnológicas, sociales y entre países. En este caso, el 80\% de los estudiantes ya estaba sensibilizado acerca de dicha cuestión, por lo que nuestra misión fue potenciarla. En el caso del alumnado restante el reto era empoderarlo a través de las redes sociales y con los medios disponibles en cada momento. Este objetivo se ha convertido en prioritario, ya que posee un impacto muy positivo hacerles ver las posibilidades de convertirse en agentes de empoderamiento y cambio en su puesto de desempeño profesional (Prieto-Jiménez, Domínguez-Fernández y López-Catalán, 2018; Maiztegui, Villardón, Navarro y Santibáñez, 2019). Todo ello se está consiguiendo mediante el enfoque metodológico y de evaluación, potenciando la dimensión de la competencia de aprender a desaprender, las teorías del decrecimiento, la educación crítica, inclusiva, emancipadora, transformadora y holística (integrando transversalmente los ejes epistemológicos fundamentales: clima, género, salud, consumo responsable, comercio justo, etc.). Consideramos que esta finalidad la estamos consiguiendo, ya que nuestros egresados manifiestan haber sufrido un cambio, tanto profesional como personal, tras su paso por el máster.

Finalmente, el tercer objetivo, relacionado con la empleabilidad, lo ubicamos desde la inserción sociolaboral y su desempeño profesional. Esta dimensión está altamente vinculada con las dos anteriores, ya que, en la medida en la que están mejor formados, concienciados y comprometidos con la EpD, sus posibilidades de trabajo aumentan. El tener un perfil más polivalente les supone el valor añadido que los diferencia de los profesionales de su mismo sector, así como la especialización en los tres perfiles indicados: educador, técnico y directivo. Esto les potencia un mayor nivel de empleabilidad y la ocupación de puestos de desempeño con mayor responsabilidad que los llevará a empoderarse y generará un mayor impacto, tanto de ellos como del máster. La alta tasa de empleabilidad del máster, así como los datos recabados, nos lleva a afirmar que en la actualidad más del $83 \%$ se encuentra trabajando. Específicamente un $80 \%$ desempeña labores de docencia o trabajos en ONGD y ocupa puestos directivos. Esto supone un mayor impacto en sus acciones (los titulados coordinan e implementan acciones y programas de EpD que no se desarrollaban anteriormente) y un efecto multiplicador en cuanto a los principios y los valores de la EpD. También es de suma relevancia ese grupo importante que realizó tesis doctorales y que actualmente se dedica a la docencia universitaria, tanto a nivel nacional como internacional. 
Por último, en base a estos datos que describimos, debemos reconocer que aún queda mucho camino por recorrer en la mejora del máster. Actualmente, en la preocupación por la adaptación al contexto, se ha puesto en marcha un proceso de modificación del título (a pesar de estar acreditados hasta el año 2022). Esta modificación ha tenido cuatro ejes fundamentales:

a) Potenciar la creación de redes con ONGD para mejorar la calidad de las prácticas como eje del currículo y la empleabilidad como impacto prioritario y de transferencia.

b) Adaptar el currículum a las grandes metas globales (ODS): módulos, materias y asignaturas.

c) Incluir metodologías abiertas, participativas y más coherentes con nuestro modelo de EpD (Domínguez-Fernandez, Prieto-Jiménez y Álvarez-Bonilla, 2019).

d) Revisar el proceso de evaluación, tanto de los módulos como del trabajo de fin de máster, integrándolos como parte de la aplicación de la teoría al análisis de la práctica de las organizaciones para la elaboración de propuestas de mejora.

Se evidencia así que el alumnado posee las competencias suficientes y está cualificado para desarrollar los tres perfiles (educador, técnico y directivo) en cualquier tipo de organización y contexto. Consideramos que este reto es la clave para el compromiso necesario que la Agenda 2030 (ONU, 2015) está requiriendo a todos los sectores sociales, y en especial en el ámbito universitario.

\section{Referencias bibliográficas}

AACID (2015). Plan Andaluz de Cooperación para el Desarrollo 2015-2018. Recuperado de $<$ https://cutt.ly/Ayh0Vpt>.

- (2018). Plan Andaluz de Cooperación para el Desarrollo 2008-2011. Recuperado de $<$ http://cort.as/-I9XT>.

AECID (2009). Plan Director de la Cooperación Española 2009-2012. Recuperado de $<$ http://cort.as/-I9Xc>.

- (2018). Plan Director de la Cooperación Española 2018-2021. Recuperado de $<$ http://cort.as/-I9Xk>.

Alonso, J.A. y Sanahuja, J.A. (2007). Un mundo en transformación: Repensar la agenda del desarrollo. En InTERmón-Oxfam. La realidad de la ayuda 2006-2007 (pp. 179-204). Barcelona: Intermón-Oxfam.

Argibay, M. y Celorio, G. (2005). La Educación para el Desarrollo. Vitoria-Gasteiz: Servicio Central de Publicaciones del Gobierno Vasco.

Bolívar, A. (2007). Educación para la Ciudadanía: Algo másque una asignatura. Barcelona: Graó.

Boni Aristizábal, A. (2010). El sistema de la cooperación internacional al desarrollo: Evolución histórica y retos actuales. En C. Calabuig y M. GómezTORREs (coord.). Cuadernos de cooperación para el desarrollo. Vol. 1, La cooperación internacional para el desarrollo. Valencia: Universidad Politécnica de Valencia.

Bustelo, P. (1998). Teorías contemporáneas del desarrollo económico. Madrid: Síntesis. 
Celorio Díaz, J.J. (2014). Educar para la ciudadanía global: Retos y desafíos a los paradigmas establecidos. En J.L. PAstoriza (ed.). Educar para la ciudadanía global: Experiencias, herramientas y discursos para el cambio social (pp. 103-109). Vigo: Fundación Isla de Couto.

Celorio, G. y López de Munain, A. (coords.) (2007). Actas del III Congreso de Educación para el Desarrollo. Bilbao: Hegoa.

Covey, S. (1998). Los 7 hábitos de la gente altamente efectiva. Barcelona: Paidós.

Domínguez-Fernández, G.; Prieto-Jiménez, E. y Álvarez-Bonilla, F.J. (2019). Las situaciones reales del docente como estrategia de aprendizaje inicial del profesorado de secundaria: El modelo SIRECA. Profesorado, 23(3), 129-149. <https://doi.org/10.30827/profesorado.v23i3.9512>

Garrote Rojas, D. y Jiménez-Fernández, S. (2018). Perfil motivacional en estudiantes del grado de Maestro en Educación Infantil y Primaria. Educar, 54(2), 351-368. <https://doi.org/10.5565/rev/educar.899>

Gómez, M. y Sanahuja, J.A. (1999). El sistema internacional de cooperación al desarrollo. Madrid: CIDEAL.

Groves, L. y Hinton, R. (2004). Inclusive aid: Changing Power and Relationship in International Development. Londres: Earthscan.

Jones, S.J. (2003). Cibersociedad 2.0: Una nueva visita a la comunidad y la comunicación mediada por ordenador. Barcelona: UOC.

«Ley 23/1998, de 7 de julio, de Cooperación Internacional para el Desarrollo». Boletín Oficial del Estado, 162, 22755-22765 (8 de julio de 1998). Recuperado de $<$ http://cort.as/-I9Y7>.

López-Roldán, P. y FACHelli, S. (2015). Metodología de Investigación Social Cuantitativa. Bellaterra: Dipòsit Digital de Documents de la Universitat Autònoma de Barcelona.

Maiztegui, C.; Villardón, L.; Navarro, M.A. y Santibáñez, R. (2019). Hacia un enfoque de justicia social: La percepción del profesorado sobre la educación intercultural en contextos de escasa presencia de alumnado extranjero. Educar, 55(1), 119-140. $<$ https://doi.org/10.5565/rev/educar.980>

Mesa, M. (2000). Generaciones de Educación para el Desarrollo. Madrid: Popular.

- (2014). La educación para la ciudadanía global en el contexto actual. En J.L. PAstoriza (ed.). Educar para la ciudadanía global: Experiencias, herramientas y discursos para el cambio social (pp. 110-121). Vigo: Fundación Isla de Couto.

Morón-Marchena, J.A.; López-Noguero, F. y Cobos-Sanchiz, D. (2017). El uso de las redes sociales en Educación para la Salud: Una experiencia de empoderamiento social en Nicaragua. Profesorado, 21(4), 439-457. Recuperado de <https:// digibug.ugr.es/handle/10481/49040>.

ONU (2015). Transformar nuestro mundo: La Agenda 2030 para el Desarrollo Sostenible. Resolución 70/01. Recuperado de <https://undocs.org/es/A/RES/70/1>.

Ortega, M.L. (2007). Estrategia de Educación para el Desarrollo de la cooperación espanola. Madrid: Ministerio de Asuntos Exteriores y de Cooperación. Recuperado de <https://www.aecid.es/Centro-Documentacion/Documentos/Planificaci\%C3\% B3n\%20estrat\%C3\%A9gica\%20por\%20sectores/estrategia_educacion_desarr.pdf>.

Observatorio Argos (2016). Estudio de la situación laboral de las personas egresadas en enseñanzas universitarias en Andalucía. Sevilla: Servicio Andaluz de Empleo.

Pérez, A. y Pérez, L. (2013). Competencias docentes en la era digital: La Formación del Pensamiento Práctico. Temas de Educación, 19(1), 67-84. Recuperado de $<$ https://revistas.userena.cl/index.php/teduacion/article/view/381>. 
Prieto-Jiménez, E.; Domínguez-Fernández, G. y López-Catalán, L. (2018). Innovando en Educación para el Desarrollo en la Universidad: Hacia un nuevo planteamiento para la Agenda 2030. En E. López-Meneses, D. Cobos-Sanchiz et al. (eds.). Experiencias pedagógicas e innovación educativa (pp. 3937-3950). Barcelona: Octaedro.

Sanahuja, J. (2001). Del interés nacional a la ciudadanía global: La ayuda al desarrollo y las transformaciones de la ciudadanía global. En J. SANAHuja y M. Gómez (eds.). La Cooperación al Desarrollo en un mundo en cambio. Madrid: Cideal.

Shulman, L. (2019). Aquellos que Entienden: Desarrollo del Conocimiento en la Enseñanza. Profesorado: Revista de Currículum y Formación del Profesorado, 23(3), 269-295.

Tashakkori, A. y Creswell, J.W. (2007). Exploring the nature of research questions in mixed methods research. Journal of Mixed Methods Research, 1, 207-211.

Teddlie, Ch. y Tashakkori, A. (2009). Foundations of mixed methods research: Integrating quantitative and qualitative approaches in the social and behavioral sciences. California: Sage Publications.

The European Higher Education Area (2009). The Bologna Declaration of 19 June 1999. Bolonia: Unión Europea. Recuperado de <http://www.eees.es/pdf/Declaracion_Bolonia.pdf $>$.

The "Maastricht Global Education Declaration». Europe-Wide Global Education Congress. Maastricht, del 15 al 17 de noviembre de 2002. Recuperado de <https:// rm.coe.int $/ 168070 \mathrm{e} 540>$.

UNESCO (2015). Educación para la ciudadanía mundial: Temas y objetivos de aprendizaje. Disponible en: <http://cort.as/-I9Y>.

VidAL, J.M. (1995). Economía mundial. Madrid: McGraw-Hill. 